\title{
A pandemic of acute respiratory distress syndrome-role of lung transplant in coronavirus disease-2019-associated respiratory failure
}

\author{
Suresh Manickavel $^{1}$ (D) Neeraj Sinha ${ }^{1}$ \\ Received: 12 May 2021 / Revised: 28 June 2021 / Accepted: 30 June 2021 / Published online: 1 September 2021 \\ (C) Indian Association of Cardiovascular-Thoracic Surgeons 2021
}

\begin{abstract}
Lung transplantation may be appropriate, and may offer benefit only to a carefully selected subset of morbidly ill patients afflicted by coronavirus disease-2019. Identifying the appropriate recipient for the allocation of scarce resources, by safely navigating through the challenges that are unique to lung transplantation for coronavirus disease-2019-associated acute respiratory distress syndrome, demands a conscientious and meticulous approach. Categorizing the respiratory failure in coronavirus disease-2019 may facilitate the process of evaluation for the purpose of transplant. The progress in rescue transplants over the past two decades has greatly improved our ability to successfully perform high-risk lung transplantation.
\end{abstract}

Keywords Lung transplantation · Acute respiratory distress syndrome · Coronavirus disease-2019 · Extracorporeal membrane oxygenation

\section{Introduction}

Severe acute respiratory syndrome coronavirus-2 (SARS$\mathrm{CoV}-2$ ) is a novel virus that caused the first human infection in 2019 and then has spread throughout the world, resulting in a pandemic of viral infections and acute respiratory distress syndrome (ARDS) [1]. The case fatality rates of the ongoing pandemic of coronavirus disease-2019 (COVID-19) were estimated to be as high as 7\% [2]. Majority of deaths were caused by respiratory failure [3]. A subset of carefully selected patients among morbidly ill COVID-19 patients could potentially be rescued safely by lung transplantation. The scope of this review is to improve our understanding on the role of lung transplant surgery in COVID-19 ARDS and pulmonary fibrosis.

\section{Categories of end-stage respiratory failure in COVID-19}

COVID-19 patients with suspected end-stage lung disease are not a homogenous population from a lung transplant standpoint. The evaluation, risk stratification, and

Suresh Manickavel

suresh.transplant@gmail.com

1 University of Miami Miller School of Medicine, Miami, FL 33101, USA management would vary significantly depending on the patient profile. End-stage respiratory failure in COVID-19 can be classified into 4 groups as shown in Table 1, based on the acuity and severity of illness for the purpose of lung transplant risk stratification and evaluation.

Transplant evaluation and risk stratification for patients in group 1 are similar to patients with chronic respiratory failure from other pulmonary diseases [4]. The key difference is the trajectory of the illness. Patients who have an end-stage respiratory failure due to chronic lung diseases generally have a gradual decline in lung function over time. However, majority of patients with post-COVID-19 lung fibrosis have a gradual improvement in symptoms and oxygen requirement. A subset of these patients might have an advanced fibrosis and irrecoverable lung injury. Such patients would have a prolonged persistence of the respiratory failure without a significant improvement in lung function. They might benefit from lung transplantation, depending on the severity of disease and quality of life impairment after confirming irreversibility of respiratory failure. The timing of evaluation is usually not pressing in this group of patients, as the deconditioning that accumulated during the acute phase of illness would improve with time, even though the lung function does not recover. A delayed evaluation and transplantation could favorably impact the outcome of transplant.

Patients in groups 2, 3, and 4 are acutely ill and, hence, the timing of evaluation and transplant is vital. The 
Table 1 Categories of respiratory failure in COVID-19

\begin{tabular}{ll}
\hline Group 1 & $\begin{array}{l}\text { Post-COVID-19 pulmonary fibrosis with extensive irrecoverable lung injury and prolonged respiratory failure on } \\
\text { low flow oxygen for more than } 3 \text { to } 6 \text { months from symptom onset }\end{array}$ \\
Group 2 & Post-COVID-19 pulmonary fibrosis with chronic respiratory failure on high flow oxygen or non-invasive ventilation \\
Group 3 & Acutely ill COVID-19 patients with ARDS on invasive mechanical ventilation \\
Group 4 & Acutely ill COVID-19 patients with ARDS on ECMO \\
\hline
\end{tabular}

possibility of patient recovering with passage of time should be balanced against the risk of developing colonization or infection with multidrug-resistant organisms and severe neuromuscular and cardiac deconditioning from prolonged critical illness. Lung transplantation is a rescue procedure in this situation and the risk for complications is higher. Perhaps patients in group 2 have the highest chance of improving, and hence, watchful waiting is a reasonable approach for them. Also, group 2 patients provide a window, where a conversation about lung transplant can be initiated and a close follow-up by transplant team performed. Aggressive strategies to optimize nutrition and deconditioning should be employed during this period. The high case fatality rate of patients in groups 3 and 4 without intervention suggests that probability of a favorable outcome is poor after 4 to 6 weeks of disease. It is appropriate to consider early referral to lung transplant team in these patients. Potential candidates in group 3 should be considered for transitioning to extracorporeal membrane oxygenation (ECMO) support as early as possible, as it may facilitate awakening and physical rehabilitation.

\section{Case fatality rate for patients with COVID-19 on life support devices}

Majority of patients with COVID-19 have mild illness. Patients with severe lung injury might require mechanical ventilation and/or ECMO. A subset of critically ill patients could recover with supportive care and medical management. However, the rest of the patients might develop irreversible injury and progressive respiratory failure resulting in death. To understand the role of lung transplant in COVID-19, it is imperative to know the clinical course of patients with severe lung injury requiring life support measures.

Lim et al. conducted a meta-analysis of 69 studies from across the globe, of patients who received invasive mechanical ventilation (IMV) for COVID-19 [5]. The study analyzed the outcome of 57,420 patients on IMV. Almost half of patients with COVID-19 receiving IMV died. Case fatality rate is substantially higher among older patients, with more than $70 \%$ of patients over 60 years of age receiving IMV dying.
A period of ECMO support might help to bridge patients until the severe lung injury recovers. However, the experience so far suggests that the eventual outcome is poor in majority of patients who received ECMO. Barbaro et al. used the data from the Extracorporeal Life Support Organization Registry to evaluate the in-hospital mortality in patients who received ECMO for COVID-19 [6]. Out of the 1035 patients included in the study, only $30 \%$ were discharged home or to an acute rehabilitation center. The in-hospital death in a time-to-event analysis, assessed at 90 days after ECMO initiation, occurred in $39 \%$ of patients with data on final disposition. A significant proportion of patients were either discharged to another hospital, remained in the intensive care unit (ICU), or transferred to long-term acute care center during the 90 days follow-up. Majority of the patients in this study needed prolonged critical care support and hospitalization.

Critically ill patients with COVID-19 continue to have dismal prognosis with high case fatality rate. Intervening this course of disease with lung transplantation might favorably impact the morbidity and mortality. To achieve a safe outcome using a resource that is limited in supply, it is vital to carefully identify the right candidate and optimal timing for the intervention.

\section{History of "rescue" lung transplantation}

The first lung transplant was performed in 1963 [7]. The outcomes were poor in the initial days of lung transplant history. Even though transplants were performed in many centers globally over the following 2 decades, majority of patients died within 30 days of transplantation [8]. Successful transplantation with good long-term survival began only in 1983 [9]. Most of the early transplants were conducted as rescue intervention in critically ill patients with severe respiratory failure. In addition to differences in surgical techniques, immunosuppression, and critical care, less than optimal candidate selection could have played a role in poor outcomes in early years of lung transplant surgery.

Currently, such rescue transplants are performed for only a small subset of patients with acute or chronic lung diseases after careful candidate selection and optimization 
[10]. Using ECMO as a bridge to lung transplant, rather than mechanical ventilation, in patients with refractory respiratory failure has shown to have better postoperative outcomes, perhaps related to the ability to stay awake and participate in rehabilitation [11]. Recent analyses suggest that the survival outcomes of rescue transplants, with ECMO as a bridge, are comparable to national average for lung transplantation in the USA [12]. However, over the years, the strategies of candidate selection and preoperative management have evolved in rescue transplants and it could have contributed to the optimization of outcomes.

The Vienna Extra Corporeal Life Support (ECLS) Program published a retrospective review of their 20 years' experience in ECMO as a bridge to lung transplantation [13]. The analysis highlights the obvious "era effect" in rescue transplants. They categorized the 120 patients into three groups based on the transplant era. Patients bridged in the third era (between 2010 and 2017) have a 1 year survival of $77.1 \%$, while it was only 34\% in the first era (between 1998 and 2004). In the third era, $34.3 \%$ of patients were bridged in "awake" state, in comparison to previous eras where patients were sedated. Hoetzenecker et al. reported a review of 71 patients who were bridged to lung transplant using ECMO with a 1 year survival of $70 \%$ [14]. The median duration of ECMO before transplant was 10 days in this study population. Twenty-five patients $(35 \%)$ were extubated while on ECMO and $37 \%$ were mobilized in this case series. Biscotti et al. reported a 1 year survival of $85 \%$ in a group of 26 patients who were awake and ambulated while on ECMO before transplantation [12].

Rescue lung transplantation has gradually evolved to a sort of semi-elective procedure, with careful patient selection, optimal timing of ECMO initiation, and methodical approach in preoperative patient management.

\section{Challenges of bridging COVID-19 respiratory failure patients to lung transplantation}

(1) Establish the presence of irrecoverable loss in lung function

Establishing the presence of significant irreversible lung injury is key before pursuing lung transplantation. The morbidity and mortality risks associated with lung transplant surgery and the long-term medical management needed to successfully maintain the graft limit the number of candidates who could benefit from this intervention. Studies so far have identified that residual radiographic findings are not uncommon after COVID-19 [15]. Majority of these changes do not have a significant physiological dysfunction [16]. Respiratory impairment due to COVID-19 pneumonia could improve over time once the acute inflammation has settled. However, depending on the extent of acute lung injury and post-infection pulmonary fibrosis, the severity of residual respiratory failure might vary [17]. Three parameters could provide us adequate information to form a safe opinion regarding irreversibility. They are the severity of respiratory failure at the time of assessment, duration since symptom onset, and the pattern and severity of radiographic findings. Patients with severe hypoxic and hypercapnic respiratory failure and radiographic evidence of fibrotic changes like honey combing, cystic airspace changes, and bronchiectasis are likely to have significant residual respiratory failure even after the inflammation subsides [18].

(2) Potential for infection transmission

Lung transplant for COVID-19-associated pulmonary fibrosis carries the theoretical risk of transmission of infection to the lung allograft and the transplant care team during surgery and in the postoperative phase. Laboratory and epidemiological studies regarding the duration of SARS-CoV-2 detection in polymerase chain reaction-reverse transcription (RT-PCR), viability of the virus, and infectiousness to contacts have been conducted and can provide guidance to safely select the candidates. So far, majority of such transplants are performed in patients who are SARS-CoV-2 negative by RT-PCR from bronchoalveolar lavage (BAL) and nasopharyngeal swab [19]. However, a significant proportion of critically ill patients have a prolonged detection of ribose nucleic acid (RNA) from respiratory specimen [20]. Even though some recovered patients continue to have SARS-CoV-2 RNA detected in their upper respiratory specimens for up to 12 weeks, a study of 285 "persistently positive" persons found no secondary infections among 790 contacts [21].

RT-PCR is a useful study to confirm the etiology of patient's illness. However, it is an incomplete surrogate to evaluate transmissibility and infectiousness. Cycle threshold (Ct) in RT-PCR is a semi-quantitative measure and provides a more granular evaluation of infectiousness, compared to the dichotomized approach of RT-PCR. The identification of replication-competent virus particles from patient specimen using viral culture is a more accurate parameter to define infectiousness. However, viral cultures are only performed in biosafety level 3 laboratories, which are limited in number.

Bullard et al. demonstrated, by analyzing 90 samples for viral replication, that the $\mathrm{Ct}$ value of $>24$ and symptom-to-test time $>8$ days had high specificity to identify a specimen that would yield a negative viral culture [22]. More evidence is mounting to suggest that likelihood of recovering replication-competent virus declines 10 days after onset of symptoms [23]. Even in 
critically ill patients, the probability of detecting infectious virus particles dropped to below 5\% after 15 days of onset of symptoms [20]. Based on these findings, the likelihood of transmission of infection to the lung allograft is low beyond 3 weeks after symptom onset. Moreover, it is less likely for a COVID-19 patient to be considered for lung transplant within 4 to 6 weeks of symptom onset.

(3) Physical deconditioning

Patients with COVID-19 ARDS-associated respiratory failure are acutely ill needing critical care management with deep sedation, prolonged ventilation, paralysis, prone ventilation, and ECMO [3]. The critical illness and the interventions performed can significantly increase the risk for cardiorespiratory and neuromuscular deconditioning. In our experience, we have noticed that majority of patients with COVID-19 ARDS have significant degree of hypoxemic failure and discontinuation of sedation or paralysis has been challenging. This situation further aggravates the burden of deconditioning. The changes could accumulate within a relatively short period of immobility [24]. Lethargy and myalgia are common symptoms during the acute phase and while recovering from COVID-19, and it can exacerbate frailty in these patients. There is a lack of understanding regarding the prevalence and extent of sarcopenia and neuropathy in severe COVID-19 illness. Frailty can have serious consequences in lung transplant surgery. It is associated with greater risk for prolonged postoperative respiratory failure and mortality [25].

(4) Multi-organ failure

COVID-19 is a multi-system disorder that can potentially have short-term and long-term sequelae. Significant dysfunction of an organ system, other than lung, is a contraindication for lung transplantation. SARS$\mathrm{CoV}-2$ virus and the associated immune dysregulation have been reported to cause multi-organ damage, including cardiac injury and acute kidney injury (AKI) [26]. Numerous studies have reported a wide incidence of AKI in COVID-19 [27]. In one study of hospitalized patients, nearly $90 \%$ of patients on the ventilator developed AKI. About $15 \%$ of hospitalized patients required renal replacement therapy in this study [28]. The cardiac dysfunction can be caused by variety of mechanisms. The virus can lead to a direct myocardial injury or precipitate damage by triggering inflammation, leading to signs of heart failure. Hypoxia and lung injury could indirectly lead to heart failure due to pulmonary hypertension and right heart dysfunction [29, 30]. Cytokine storm associated with severe COVID-19 can result in endothelial cell death, vascular thrombosis, and disseminated intravascular coagulation [31].
(5) Surgical risk

Autopsy studies and explant reports during lung transplant surgeries have revealed that severe COVID19 is associated with extensive mediastinal and pleural inflammation and adhesions [19, 32]. These changes would significantly increase the risk of intraoperative bleeding and massive blood transfusion requirement. The prevalence of pulmonary hypertension is high in this group of patients and it could further escalate the burden of bleeding complications [33]. Patients needing ECMO or mechanical ventilation bridge to lung transplant have greater operative morbidity and mortality risk $[10,14]$.

(6) Informed decision-making

The successful outcome from lung transplantation requires a life-long commitment from the recipient [4, 34]. After transplant surgery, a long-term medical management with immunosuppression, infection prophylaxis, and close surveillance is needed for graft and patient survival. Potential postoperative complications, including prolonged respiratory failure, prolonged hospitalization, renal failure, stroke, and physical deconditioning, can contribute to significant morbidity [35]. The outpatient evaluation of patients with chronic lung diseases gives recipients adequate time to process the information and allows for an informed consent for the process of transplantation. However, there can be significant limitation in informed decision-making process in acutely ill patients undergoing lung transplantation.

(7) Risk of nosocomial spread of infection from recipient

There are broader implications of undertaking COVID-19 lung transplant that could extend beyond the recipient management. There is a degree of uncertainty in the risk of cross transmission within the hospital from transplant recipient reinfection and viral shedding in the immunosuppressed state. The authors believe that the risk is small, considering the time from diagnosis and the establishment of SARS-CoV-2-specific immunity in the recipient. The time demands for stringent infection control measures would compete with the time demands for meticulous assessment and care requirements for an immediate postoperative patient.

\section{Experience so far in post-COVID-19 lung transplant}

The preliminary understanding regarding the safety and feasibility of lung transplantation in COVID-19 emerges from limited experience with transplants in ARDS. Han et al. and Chen et al. published the initial reports on lung transplantation in COVID-19 from China [36, 37]. The reports 
Table 2 Initial reports on lung transplants in COVID-19

\begin{tabular}{|c|c|c|c|c|c|c|c|}
\hline First author & $\begin{array}{l}\text { No. of } \\
\text { patients }\end{array}$ & Age & Bridging strategy & Days on ECMO & $\begin{array}{l}\text { Days from } \\
\text { diagnosis to } \\
\text { transplant }\end{array}$ & $\begin{array}{l}\text { Days of } \\
\text { follow-up } \\
\text { after surgery }\end{array}$ & Mortality \\
\hline Weili Han [36] & 2 & 66,70 & ECMO and mechanical ventilation & $13 ; 12$ & $28 ; 34$ & $5 ; 2$ & 0 \\
\hline Jing-Yu Chen [37] & 3 & $66,58,73$ & ECMO and mechanical ventilation & $15 ; 7 ; 19$ & $35 ; 33 ; 38$ & $\begin{array}{l}\text { Death on day } \\
1 ; 22 ; 12\end{array}$ & 1 \\
\hline
\end{tabular}

were preliminary with only a limited follow-up as shown in Table 2. All 5 patients in these 2 reports were supported on ECMO and mechanical ventilation prior to transplant. The patients were SARS-CoV-2 PCR negative at the time of transplantation. The average number of days on ECMO prior to transplant was 13.2 days. One patient in the case series died in the operating room due to ventricular fibrillation and other complications. Intraoperative challenges including bleeding and chest cavity shrinkage were reported among the recipients.

Bharat et al. published the first case series of three consecutive patients with COVID-19 who underwent bilateral lung transplantation from the USA [19]. The surgical complexity was high in all recipients with loss of mediastinal tissue planes, extensive pleural inflammation, vascular and pleural adhesions, and reactive lymphadenopathy. All patients were on prolonged support with ECMO and mechanical ventilation before transplant. Two patients were awake and participated in physical rehabilitation before being considered for lung transplantation. Recipients were negative for SARS-CoV-2 RNA by PCR from bronchoalveolar lavage fluid prior to transplant surgery. All three recipients survived to discharge and were stable on room air at 5 months, 4 months, and 3 months follow-up.

Lang et al. published a report of lung transplantation in COVID-19-associated ARDS in a PCR-positive patient [38]. The recipient was repeatedly positive in RT-PCR testing for SARS-CoV-2 from nasopharyngeal swabs and in bronchoalveolar samples, even up to 53 days since the initial positive testing. However, the RT-PCR cycle threshold were high with values greater than 33 . Vero cell culture was used, which turned to be negative for viable virus particles. The patient was supported for 45 days on ECMO prior to transplant. Prolonged ICU stays and neuromuscular deconditioning complicated the postoperative course.

Hawkins et al. performed a review of all cases of lung transplantations that were conducted in patients with respiratory failure due to COVID-19 and published before December 15, 2020 [39]. The review had 21 patients, out of which 17 patients were transplanted using ECMO as bridging strategy. Transplants were conducted on an average of 71 days after hospitalization. The overall postoperative mortality was low in this series with only 2 patients reported to have died.
However, the presence of publication bias against reporting of poor outcomes should be taken into consideration.

A twelve-patient case series of lung transplant in COVID19-associated ARDS from six high-volume transplant centers around the world also highlighted the technically challenging nature of the surgery and feasibility of good early post-transplant outcomes [40].

\section{Conclusion}

Patients with severe respiratory failure due to COVID-19 have heterogeneous clinical characteristics. Categorizing these patients will further refine the process of candidate selection. Rescue transplantation is associated with greater resource utilization and higher risk for procedurerelated morbidity and mortality. A majority of patients with COVID-19 ARDS do not benefit from lung transplantation due to the presence of clinical factors that might prohibitively increase the risk for complications. There are challenges that are unique to lung transplant surgery for COVID-19 ARDS. Uncertainty still exists with regard to the appropriate timing of ECMO placement and transplantation. There is an unpredictable and heightened surgical risk due to pulmonary hypertension, pleural and mediastinal adhesions, and frailty. Organ allocation to such procedures involves the risk of prolonging the wait-time period, worsening the wait-list mortality rates, and quality of life measures among patients with other lung diseases. With careful patient selection and appropriate preoperative management, the outcomes of transplant surgery in COVID-19-associated respiratory failure could be comparable to current standards in lung transplantation.

Funding Nil.

\section{Declarations}

Ethics approval Not applicable as this is an opinion article with review of literature.

Informed consent Not applicable as no patients were involved. 
Statement of human and animal rights Not applicable as no experiments were performed.

Conflict of interest The authors declare no competing interests.

\section{References}

1. World Health Organization. WHO coronavirus disease dashboard. WHO Int. 2021.

2. Onder G, Rezza G, Brusaferro S. Case-fatality rate and characteristics of patients dying in relation to COVID-19 in Italy". JAMA. 2020;323:1775-6. https://doi.org/10.1001/jama.2020.4683.

3. Guan W-J, Ni Z-Y, Hu Y, et al. Clinical characteristics of coronavirus disease 2019 in China. N Engl J Med. 2020;382:1708-20. https://doi.org/10.1056/NEJMoa2002032.

4. Weill D, Benden C, Corris PA, et al. A consensus document for the selection of lung transplant candidates: 2014 - an update from the Pulmonary Transplantation Council of the International Society for Heart and Lung Transplantation. J Heart Lung Transplant. 2015;34:1-15. https://doi.org/10.1016/j.healun.2014.06.014.

5. Lim ZJ, Subramaniam A, Ponnapa Reddy M, et al. Case fatality rates for patients with COVID-19 requiring invasive mechanical ventilation. A meta-analysis. Am J Respir Crit Care Med. 2021;203:54-66. https://doi.org/10.1164/rccm.202006-2405OC.

6. Barbaro RP, MacLaren G, Boonstra PS, et al. Extracorporeal membrane oxygenation support in COVID-19: an international cohort study of the Extracorporeal Life Support Organization registry. Lancet. 2020;396:1071-8. https://doi.org/10.1016/S01406736(20)32008-0.

7. Hardy JD, Webb WR, Dalton ML, Walker GR. Lung homotransplantation in man. Transplantation. 1964. https://doi.org/10.1097/ 00007890-196411000-00039.

8. Kotloff RM, Thabut G. Lung transplantation. Am J Respir Crit Care Med. 2011;184:159-71. https://doi.org/10.1164/rccm. 201101-0134CI.

9. Toronto Lung Transplant Group. Unilateral lung transplantation for pulmonary fibrosis. N Engl J Med. 1986;314-1140-5. https:// doi.org/10.1056/NEJM198605013141802.

10. Sunder T. Extracorporeal membrane oxygenation and lung transplantation. Indian J Thorac Cardiovasc Surg. 2021;37:327-37. https://doi.org/10.1007/s12055-020-01099-5.

11. Kurihara C, Walter JM, Singer BD, et al. Extracorporeal membrane oxygenation can successfully support patients with severe acute respiratory distress syndrome in lieu of mechanical ventilation. Crit Care Med. 2018;46:e1070-3. https://doi.org/10.1097/ CCM.0000000000003354.

12. Biscotti M, Gannon WD, Agerstrand C, et al. Extracorporeal membrane oxygenation as a bridge to lung transplantation based on intent to treat: a single-center experience. J Heart Lung Transplant. 2015. https://doi.org/10.1016/j.healun.2015.01.025.

13. Benazzo A, Schwarz S, Frommlet F, et al. Twenty-year experience with extracorporeal life support as bridge to lung transplantation. J Thorac Cardiovasc Surg. 2019. https://doi.org/10. 1016/j.jtcvs.2019.02.048.

14. Hoetzenecker K, Donahoe L, Yeung JC, et al. Extracorporeal life support as a bridge to lung transplantation-experience of a high-volume transplant center. J Thorac Cardiovasc Surg. 2018. https://doi.org/10.1016/j.jtcvs.2017.09.161.

15. Han X, Fan Y, Alwalid O, et al. Six-month follow-up chest CT findings after severe COVID-19 pneumonia. Radiology. 2021;299:E177-86. https://doi.org/10.1148/radiol.2021203153.

16. Frija-Masson J, Debray M-P, Gilbert M, et al. Functional characteristics of patients with SARS-CoV-2 pneumonia at 30 days post-infection. Eur Respir J. 2020. https://doi.org/10.1183/ 13993003.01754-2020.

17. Zhao Y-M, Shang Y-M, Song W-B, et al. Follow-up study of the pulmonary function and related physiological characteristics of COVID-19 survivors three months after recovery. EClinicalMedicine. 2020. https://doi.org/10.1016/j.eclinm.2020.100463.

18. Rai DK, Sharma P, Kumar R. Post covid 19 pulmonary fibrosisis it real threat? Indian J Tuberc. 2021;68:330-3. https://doi.org/ 10.1016/j.ijtb.2020.11.003.

19. Bharat A, Querrey M, Markov NS, et al. Lung transplantation for patients with severe COVID-19. Sci Transl Med. 2020. https://doi.org/10.1126/scitranslmed.abe4282.

20. Li T-Z, Cao Z-H, Chen Y, et al. Duration of SARS-CoV-2 RNA shedding and factors associated with prolonged viral shedding in patients with COVID-19. J Med Virol. 2021;93:506-12. https://doi.org/10.1002/jmv.26280.

21. Korea Centers for Disease Control and Prevention. Findings from investigation and analysis of re-positive cases. 2020. [Online]. Available: https://www.cdc.go.kr/board/board.es? $\mathrm{mid}=\mathrm{a} 30402000000 \& \mathrm{bid}=0030 \& \mathrm{act}=$ view $\&$ list $\_$no $=36726$ $7 \&$ nPage $=1$ externalicon. Accessed 19 May 2020.

22. Bullard J, Dust K, Funk D, et al. Predicting infectious SARSCoV-2 from diagnostic samples. Clin Infect Dis. 2020. https:// doi.org/10.1093/cid/ciaa638.

23. Discontinuation of Transmission-Based Precautions and Disposition of Patients with SARS-CoV-2 Infection in Healthcare Settings. https://www.cdc.gov/coronavirus/2019-ncov/hcp/dispo sition-hospitalized-patients.html

24. Carda S, Invernizzi M, Bavikatte G, et al. COVID-19 pandemic. What should physical and rehabilitaion medicine specialists do? A clinician's perspective. Eur J Phys Rehabil Med. 2020;56:515-24. https://doi.org/10.23736/S1973-9087.20. 06317-0.

25. Casey P, Ang Y, Sultan J. COVID-19-induced sarcopenia and physical deconditioning may require reassessment of surgical risk for patients with cancer. World J Surg Onc. 2021;19:8. https://doi.org/10.1186/s12957-020-02117-x.

26. Noris M, Benigni A, Remuzzi G. The case of complement activation in COVID-19 multiorgan impact. Kidney Int. 2020;98:31422. https://doi.org/10.1016/j.kint.2020.05.013.

27. Kunutsor SK, Laukkanen JA. Renal complications in COVID-19: a systematic review and meta-analysis. Ann Med. 2020;52:34553. https://doi.org/10.1080/07853890.2020.1790643.

28. Hirsch JS, Ng JH, Ross DW, et al. Acute kidney injury in patients hospitalized with COVID-19. Kidney Int. 2020;98:209-18. https://doi.org/10.1016/j.kint.2020.05.006.

29. Puntmann VO, Carerj ML, Wieters I, et al. Outcomes of cardiovascular magnetic resonance imaging in patients recently recovered from coronavirus disease 2019 (COVID-19)". JAMA Cardiol. 2020;5:1265-73. https://doi.org/10.1001/jamacardio.2020. 3557.

30. Inciardi RM, Lupi L, Zaccone G, et al. Cardiac involvement in a patient with coronavirus disease 2019 (COVID-19)". JAMA Cardiol. 2020;5:819-24. https://doi.org/10.1001/jamacardio.2020. 1096.

31. Fajgenbaum DC, June CH. Cytokine storm. N Engl J Med. 2020;383:2255-73. https://doi.org/10.1056/NEJMra2026131.

32. Ackermann M, Verleden SE, Kuehnel M, et al. Pulmonary vascular endothelialitis, thrombosis, and angiogenesis in Covid-19. N Engl J Med. 2020;383:120-8. https://doi.org/10.1056/NEJMo a2015432.

33. Pagnesi M, Baldetti L, Beneduce A, et al. Pulmonary hypertension and right ventricular involvement in hospitalised patients with COVID-19. Heart. 2020;106:1324-31. https://doi.org/10.1136/ heartjnl-2020-317355. 
34. Sunder T, Ramesh TP, Kumar KM, Suresh M, Singh SP, Seth S. Lung transplant: the Indian experience and suggested guidelines - part 1 selection of the donor and recipient. J Pract Cardiovasc Sci. 2018;4:88-95. https://doi.org/10.4103/jpcs.jpcs_46_18.

35. Chan EG, Bianco V III, Richards T, et al. The ripple effect of a complication in lung transplantation: evidence for increased longterm survival risk. J Thorac Cardiovasc Surg. 2016;151:1171-80. https://doi.org/10.1016/j.jtcvs.2015.11.058.

36. Han W, Zhu M, Chen J, et al. Lung transplantation for elderly patients with end-stage COVID-19 pneumonia. Ann Surg. 2020;272:e33-4. https://doi.org/10.1097/SLA.0000000000 003955.

37. Chen J-Y, Qiao K, Liu F, et al. Lung transplantation as therapeutic option in acute respiratory distress syndrome for COVID-2019-related pulmonary fibrosis. Chin Med J (Engl). 2020. https://doi.org/ $10.1097 / \mathrm{cm} 9.0000000000000839$.

38. Lang $\mathrm{C}$, Jaksch $\mathrm{P}$, Hoda MA, et al. Lung transplantation for COVID-19-associated acute respiratory distress syndrome in a PCR-positive patient. Lancet Respir Med. 2020;8:1057-60. https://doi.org/10.1016/S2213-2600(20)30361-1.

39. Hawkins RB, Mehaffey JH, et al. Lung transplantation for severe post-coronavirus disease 2019 respiratory failure. Transplantation. 2021;105:1381-7. https://doi.org/10.1097/tp.0000000000003706.

40. Bharat A, Machuca TN, Querrey M, et al. Early outcomes after lung transplantation for severe COVID-19: a series of the first consecutive cases from four countries. Lancet Respir Med. 2021;9:487-97. https://doi.org/10.1016/S2213-2600(21)00077-1.

Publisher's note Springer Nature remains neutral with regard to jurisdictional claims in published maps and institutional affiliations. 